\title{
A COMPARISON OF OPTIMIZATION TECHNIQUES FOR POWER PATTERNS WITH LOW SIDELOBES GENERATED BY LINEAR ARRAYS WITH EFFICIENT EXCITATION DISTRIBUTIONS
}

\author{
S. Kyle Smith ${ }^{1}$ (Student Member, IEEE), Julio C. Brégains ${ }^{2}$ (Student Member, IEEE), \\ Kathleen L. Melde ${ }^{1}$ (Senior Member, IEEE), and Francisco Ares ${ }^{2}$ (Senior Member, IEEE) \\ ${ }^{1}$ Dept. of ECE, University of Arizona, Tucson, AZ 85721, USA \\ ${ }^{2}$ Radiating Systems Group, Department of Applied Physics, \\ Univ. of Santiago de Compostela \\ Campus Sur, 15782, Santiago de Compostela, Spain \\ Email: faares@usc.es
}

\begin{abstract}
Equispaced linear arrays that have a uniformly excited central elements and monotonically decreasing flanking segments have been shown to yield low sidelobe patterns with specified constraints on the effective radiated voltage (ERV). An analytical method for generating these distributions using a constrained least squares (CLS) method given restrictions on both the peak amplitude of the elements and the ERV has been discussed in an earlier work. Simulated annealing (SA) is a versatile global optimization technique that can be effectively used for similar purposes, but providing additional control over some other design parameters. In this paper we inspect both methods -indicating some of their advantages and drawbacks- through some numerical results.
\end{abstract}

\section{INTRODUCTION}

Linear arrays uniformly excited are very desirable, since their feedings are easily adjusted and, which is the most important characteristic, the arrays fed in that manner have the maximum available efficiency, in terms of power radiated to the space. Nevertheless, such a distribution does not allow the convenient control and exploitation of the sidelobe topography. In an earlier work [1] it has been demonstrated that a constrained least squares (CLS) method applied to the synthesis of linear arrays with some restrictions on the excitation amplitudes of the elements provides a useful tool for antenna designers, leading to a distribution that possesses a central segment uniformly excited and a monotonic decay toward the edges. This method, whose foundations can be found in an earlier work written by Iglehart [2], applies restrictions on both the peak amplitude of the elements and the effective voltage ratio ( $E R V$, a coefficient that corresponds to the mean value of the amplitude distribution). Furthermore, the CLS method can be specified so as to minimize the average energy measured from 
the first (or the second, in accordance to the specifications of the antenna designer) null in a symmetric sum diagram [1].

On the other hand, the simulated annealing (SA) is a versatile global optimization technique that has been effectively used for a wide variety of antenna array applications [3, 4]. In principle, one should be able to perform a SA program for linear arrays and provide a suitable cost function in order to generate the same results as the CLS method. Although SA is, in general, a slow algorithm (in terms of computation time), its main advantage resides on the number and diversity of parameters that it can control, by simply inserting the corresponding term in the cost function that the algorithm manages. As a summary, it can be stated that the analytical CLS approach solves only a few specific problems, but very efficiently, whereas SA technique can solve a variety of related problems and also obtain complex sidelobe topographies [3, 4]. In this paper we compare the results obtained from generating radiation patterns for linear arrays with high amplitude distribution efficiency and low sidelobes using the CLS and SA techniques.

\section{DESCRIPTION OF THE METHODS.}

\subsection{CONSTRAINED LEAST SQUARES METHOD.}

Consider a discrete array with an even number $(2 \mathrm{~N})$ of isotropic elements located along the z-axis, with a real-valued excitation distribution given by $g\left(z_{n}\right), n \in[1,2 N]$. The array factor of this arrangement can be calculated through the usual expression:

$$
F(\theta)=\sum_{n=1}^{2 N} g\left(z_{n}\right) e^{j \beta z_{n} \cos \theta}
$$

where $\beta=2 \pi / \lambda$ is the wavenumber, $z_{n}$ is the position of the $n$-th element, $\lambda$ is the wavelength, and $\theta$ is the angle measured from the array axis.

Consider further that the array and the excitation distribution are symmetric around the array center located at $z=0$. The CLS method can be applied by minimizing the weighted mean square value (i. e. the energy radiated by the array) of eq. (1), as explained in [1], in a specified sidelobe region (from first or second null until the end of the pattern radiation zone), subject to the following constraints: 


$$
\begin{aligned}
& \left|g\left(z_{n}\right)\right|=\left\{\begin{array}{l}
\leq 1, \text { if } n \in[1,2 N] \\
0, \text { otherwise }
\end{array}\right. \\
& E R V=\frac{1}{2 N} \sum_{n=1}^{2 N} g\left(z_{n}\right)=\frac{1}{N} \sum_{n=1}^{N} g\left(z_{n}\right)
\end{aligned}
$$

When $E R V=1$, the distribution is uniform, when $E R V<1$, the distribution obtained with the method will be tapered. A proof that this excitation distribution will be optimal is given in an analogous work applied to filter theory [5].

In this work, the CLS method has been implemented to minimize sidelobe energy beyond the second null. Improved results are obtained by the use of an iterative approach which will now be described. It is unfeasible to know the exact location of the second null prior to running the CLS simulation. An approximation to this value can be made using uniform distribution equations; however, this is not very accurate due to null shifting that occurs when the distribution is tapered. This causes discrepancy between the initial approximation and the actual location of the second null in the resulting pattern. The iterative CLS approach can resolve this discrepancy. This approach begins with the same initial approximation obtained from uniform distribution formulas, however, the observed location of the resulting null in the CLS pattern is reentered as the new optimization angle for each subsequent iteration. After several iterations, the initial minimization angle and the actual location of the second null in the resulting pattern agree, and significantly improved results are so obtained.

\subsection{SIMULATED ANNEALING METHOD.}

In the SA approach, a cost function is minimized to achieve the desired values of the power pattern calculated with the expression (1). A general cost function obtained with the proper choice of $c_{i}$ (weights) is given by:

$$
C=c_{1}\left(E R V_{o}-E R V_{d}\right)^{2}+c_{2} H\left(S L L_{\max , o}, S L L_{\text {max }, d}\right)+c_{3}(1 / D)+c_{4} S L L_{a v}
$$

The subscripts $o$, and $d$, max and $a v$ indicate obtained, desired, maximum and average values, respectively. $E R V$ has the same significance as that given in (2), and $H$ is the Heaviside step function defined as:

$$
H\left(S L L_{\max , o}, S L L_{\max , d}\right)=\left\{\begin{array}{l}
1, \text { if } S L L_{\max , o}>S L L_{\max , d} \\
0, \text { otherwise }
\end{array}\right.
$$


$S L L$ are the sidelobe levels (the maxima given in $\mathrm{dBs}$, whereas the average as its absolute value relative to the peak of the main beam), whereas $D$ corresponds to peak directivity.

First, an array distribution is computed by taking uniformly spaced points of a continuous Taylor distribution and then choosing the values of the extremes to coincide with the first and last elements of the array. SA is then used to perturb the values of its roots so as to minimize the cost function given by (3). The values given to the cost function weights will produce different optimal patterns and distributions.

\section{COMPARISON OF RESULTS}

Consider a linear array of 30 elements a distance $0.5 \lambda$ apart. The CLS method and two different SA cases were used to compute far-field patterns with $E R V=0.9,0.8$, and 0.7. In the CLS approach, the energy beyond the second sidelobe is minimized for a fixed $E R V$. In the first $\mathrm{SA}$ approach $\left(\mathrm{SA}_{1}\right)$, the $\mathrm{c}_{4}$ weight, which controls the average sidelobe level, was set to 0 and the other weights were set to 10,1 , and $0.5\left(c_{1}, c_{2}\right.$ and $c_{3}$, respectively). This cost function maximizes directivity for a fixed $E R V$ and a specified peak sidelobe level. The desired peak sidelobe level was set to $-17 \mathrm{~dB}$ for $E R V_{d}=0.9,-20 \mathrm{~dB}$ for $E R V_{d}=0.8$, and $-25 \mathrm{~dB}$ for $E R V_{d}=0.7$. In the second SA approach $\left(\mathrm{SA}_{2}\right)$, the $c_{1}$ weight was set to 1 , the $c_{4}$ weight was set to 4.5 and the other weights were set to zero. This cost function minimizes the overall average sidelobe level for a fixed $E R V_{d}$. Table 1 compares the pattern statistics for these cases. For brevity, only patterns for $E R V=0.9$ are shown in Figures $1-3$ (CLS, $\mathrm{SA}_{1}$, and $\mathrm{SA}_{2}$ cases). The excitation amplitudes are represented in figure 4 . As can be seen from table 1 , the $\mathrm{SA}_{1}$ distribution yields the highest directivity and the highest average sidelobe levels. The CLS and $\mathrm{SA}_{2}$ cases distributions show comparable values for directivity, peak sidelobe level, and average sidelobe level. For $E R V=0.7$ and 0.8 the peak sidelobe level is highest for the CLS case. The first sidelobe is excluded in the minimization of sidelobe energy in the CLS approach, while the $\mathrm{SA}_{2}$ approach includes the first sidelobe in the minimization of the average sidelobe level. The CLS and $\mathrm{SA}_{2}$ distributions are in excellent agreement and have a monotonic taper beyond the uniform region. The $\mathrm{SA}_{1}$ distributions have the slight increase at the ends that are characteristic of Taylor distributions with high $\bar{n}$ values (in all examples performed here, $\bar{n}=8$ was taken when using any of the SA methods).

\section{CONCLUDING REMARKS}

The results show that with the appropriate terms (and/or weights) in the cost function, the SA approach can lead to CLS-like patterns and distributions. These results also provide an interesting numerical validation of the 
forms of the distribution functions derived in [2] and [5]. The advantage of SA techniques is the ability to provide variations to the CLS method for differing sidelobe topographies. The edge brightening appeared in the amplitude distributions obtained with the SA approach could be reduced by adding a corresponding term in the cost function (3), penalizing that behavior. Nevertheless, in this sense it is of concern the implementation of other kind of excitation distributions, analogous to Taylor's, as, for example, one appeared in a work elaborated by Rhodes [6]. In that case, the amplitude distributions behave very similarly to those obtained with the CLS implementation, with a central segment and flanking monotonic decaying curves, as was verified by the authors by the inspection of some results not shown here for brevity. As straightforward extensions of the methods, circular continuous sources and $\phi$-symmetrical arrays are under consideration by part of the authors in a current research [7].

\section{ACKNOWLEDGEMENTS}

This work was supported in part by the National Science Foundation NSF Grant ECS-0098547 and by the Spanish Ministry of Science and Technology under project TIC2002-04084-C03-02.

\section{REFERENCES}

[1] Virga, K. L., and Taylor, M. L., "Transmit Patterns for Active Linear Arrays with Peak Amplitude and Radiated Voltage Distribution Constraints”, IEEE Trans. on Antennas and Propagat., Vol. 49, No. 5, pp. 732$739,2001$.

[2] S. C. Iglehart, "Design of Weighting Functions under Peak Amplitude and Effective Radiated Voltage Constraints," IEEE Trans. on Aerospace and Electronic Systems, pp 267-274, 1979.

[3] J. A. Rodríguez, F. Ares, E. Moreno, "Sum Pattern Side Lobe Minimization for Linear Arrays with a Uniformly Excited Central Segment”, Journal of Electromagnetics Waves and Applications, pp. 409-417, 2003.

[4] F. Ares, "Application of Genetic Algorithms and Simulated Annealing to Some Antenna Problems", from the book "Electromagnetic Optimization by Genetic Algorithms", Edited by Y. Rahmat-Samii, and E. Michielssen, John Wiley \& Sons, chapter five, 1999.

[5] A. D. Shnidman, "Solution to a Class of Optimization Problems with Amplitude Constraints", IEEE Trans. on Comm., Vol. 23, No. 9, pp. 979-983, 1975. 
[6] D. R. Rhodes, "On the Taylor Distribution", IEEE Trans. on Antennas and Propagat., Vol. 20, No 2, pp. 143$145,1972$.

[7] J. C. Brégains, F. Ares, S. K. Smith and K. Melde, "Control de la Topografía de Lóbulos Laterales de Patrones en Potencia Tipo Suma Generados por Distribuciones Circulares Continuas de Elevada Eficiencia”, to be presented in September 2004, at the XIX Simposium Nacional de la Unión Científica Internacional de Radio, URSI 2004, in Barcelona, Spain. 


\section{LEGENDS FOR FIGURES AND TABLES}

TABLE 1. Comparison table of the results obtained with the CLS and SA methods.

FIGURE 1. Power pattern obtained with CLS method, establishing $E R V=0.9$.

FIGURE 2. Power pattern obtained with $\mathrm{SA}_{1}$ method, establishing $E R V_{d}=0.9$ in the cost function (3).

FIGURE 3. Power pattern obtained with $\mathrm{SA}_{2}$ method, establishing $E R V_{d}=0.9$ in the cost function (3).

FIGURE 4. Comparison of CLS and SA distributions for $E R V=0.9$ that generate the power patterns shown in figures 1-3. 
TABLE 1

\begin{tabular}{|c|c|c|c|c|c|}
\hline METHOD & ERV & D & $\begin{array}{l}\text { Peak SLL } \\
\text { (dB) }\end{array}$ & $\underset{\text { (dB) }}{\operatorname{Avg.~SLL~}}$ & $\begin{array}{l}\text { Avg. SLL (dB) } \\
\text { Excluding 1 } 1^{\text {st }} \\
\text { Sidelobe }\end{array}$ \\
\hline $\mathrm{SA}_{1}$ & \multirow{3}{*}{0.9} & 29.24 & -17.00 & -24.70 & -25.30 \\
\hline $\mathrm{SA}_{2}$ & & 28.80 & -14.24 & -27.21 & -33.42 \\
\hline CLS & & 28.89 & -14.47 & -27.37 & -33.11 \\
\hline $\mathrm{SA}_{1}$ & \multirow{3}{*}{0.8} & 28.73 & -20.09 & -28.52 & -29.29 \\
\hline $\mathrm{SA}_{2}$ & & 27.55 & -17.47 & -30.51 & -36.39 \\
\hline CLS & & 26.92 & -16.43 & -29.79 & -40.10 \\
\hline $\mathrm{SA}_{1}$ & \multirow{3}{*}{0.7} & 27.26 & -25.00 & -30.61 & -30.65 \\
\hline $\mathrm{SA}_{2}$ & & 25.78 & -23.64 & -35.98 & -39.39 \\
\hline CLS & & 25.41 & -18.86 & -32.12 & -50.24 \\
\hline
\end{tabular}




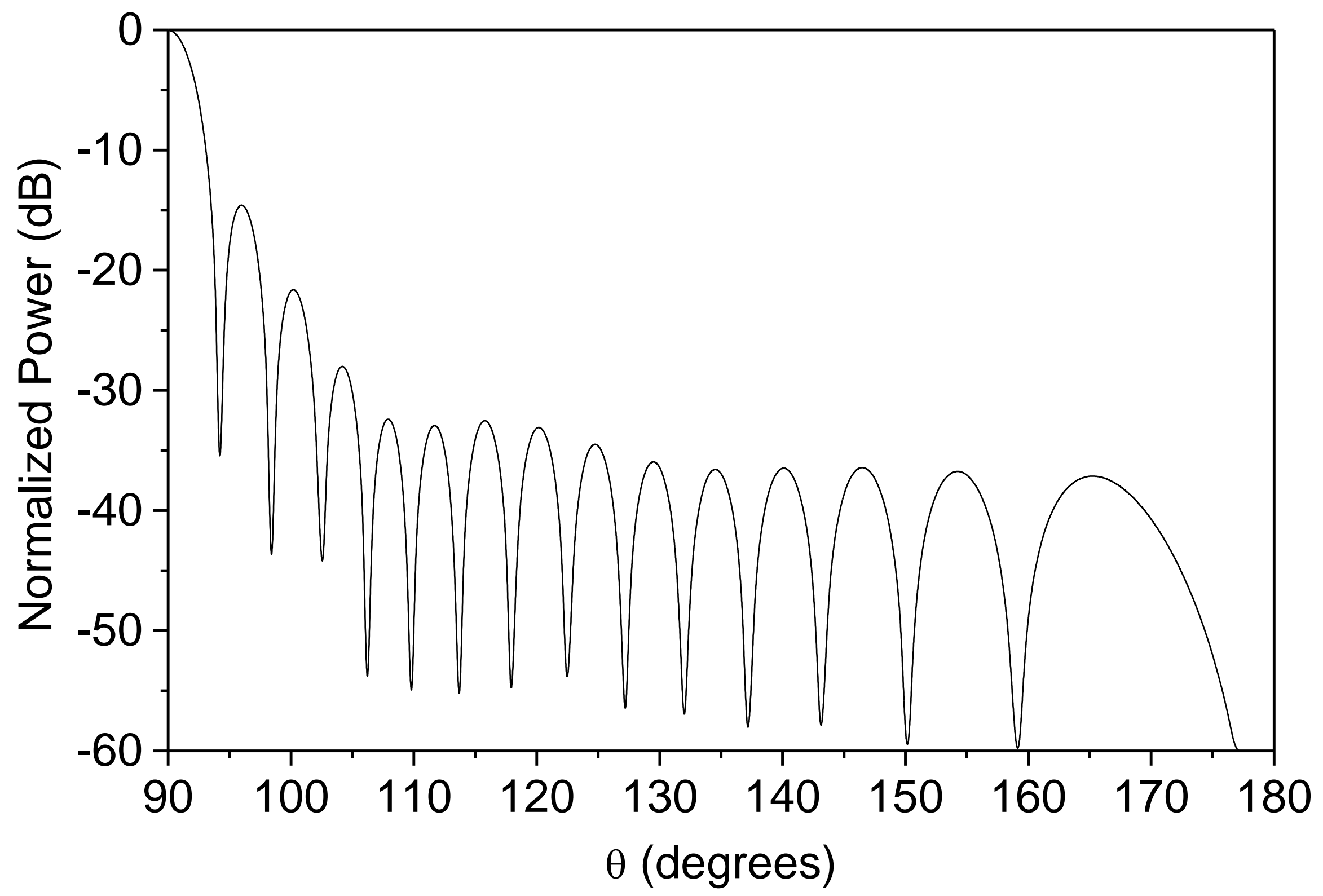

Fig. 1 


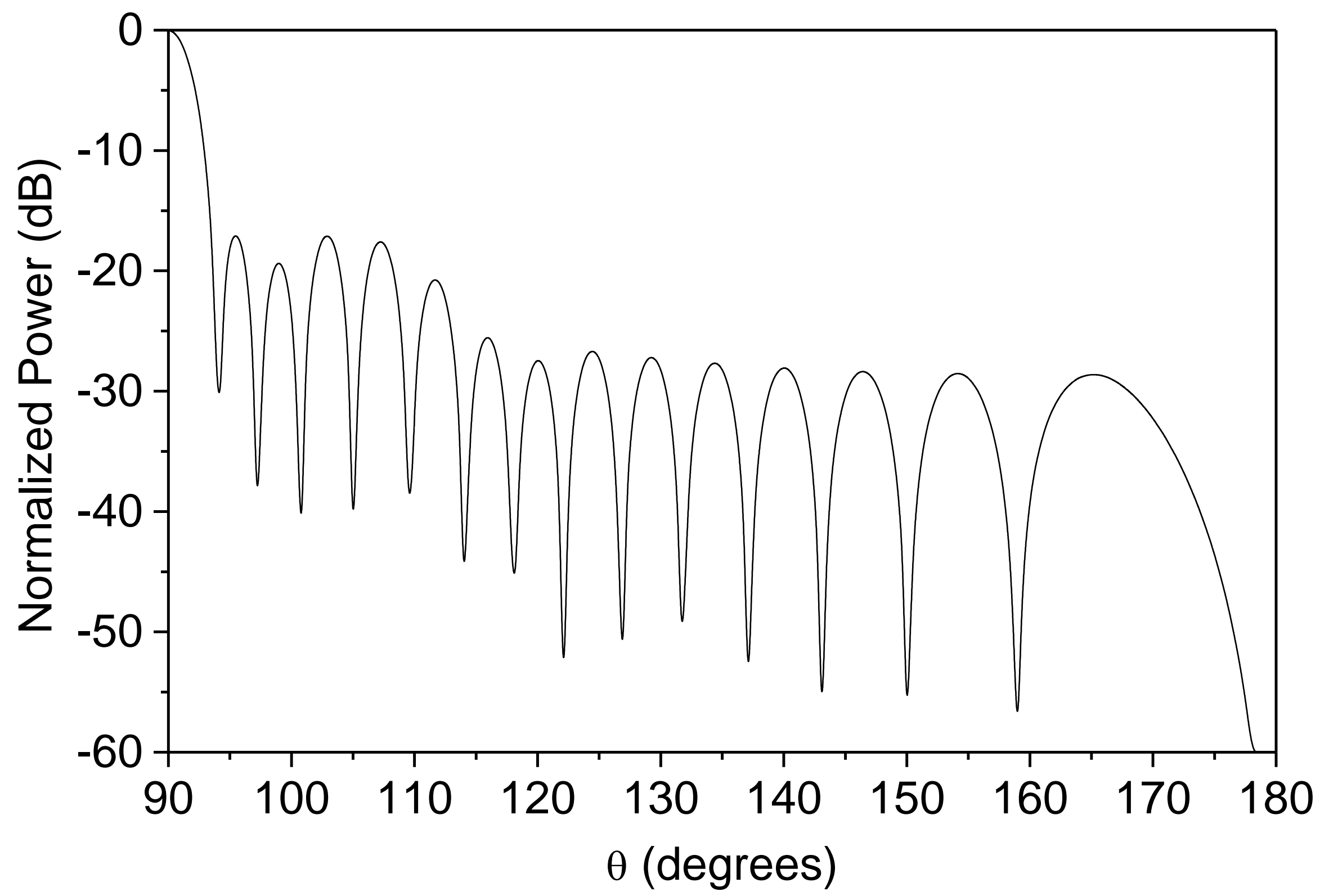

Fig. 2 




Fig. 3 


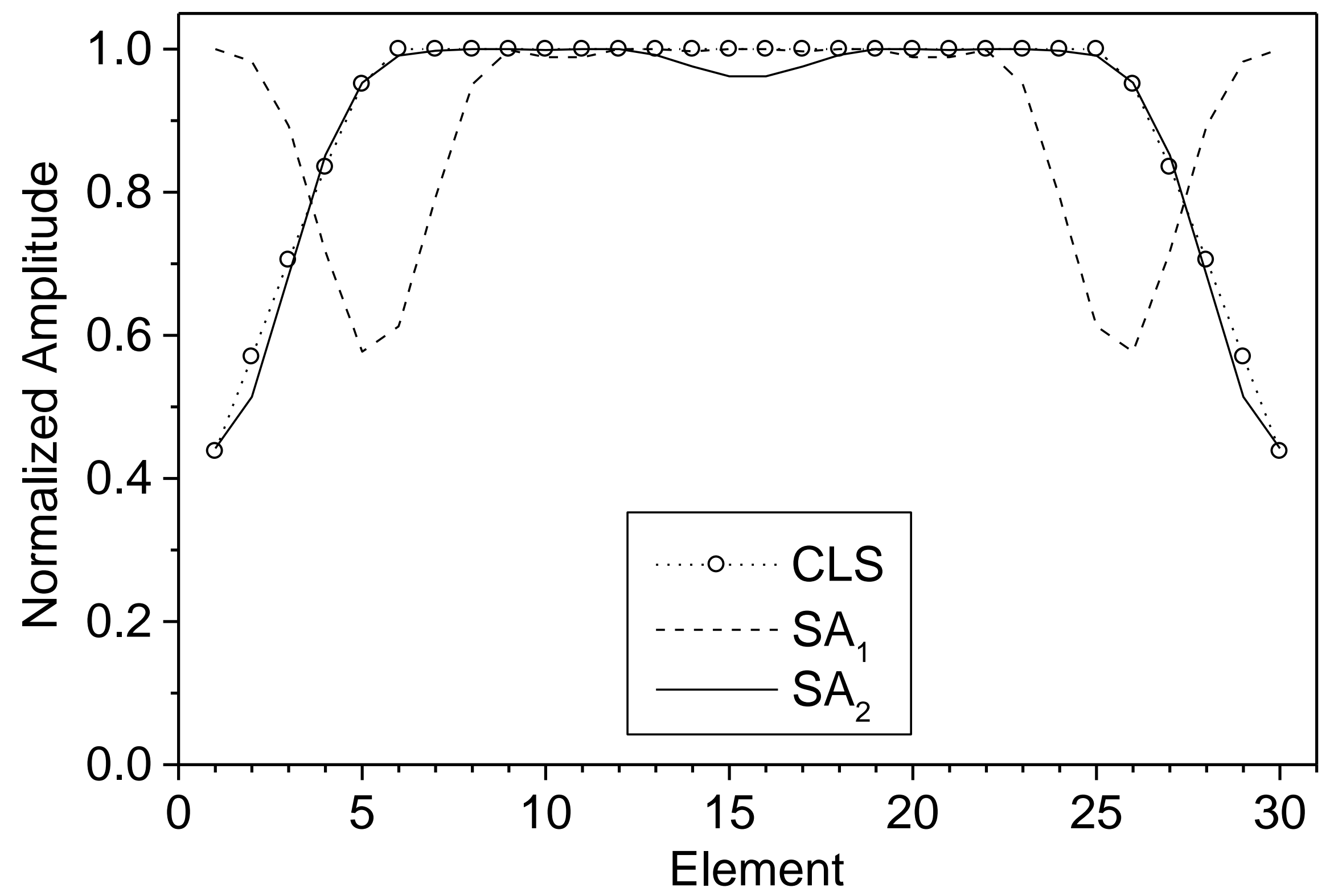

Fig. 4 